\title{
Clinical Prognostic Factors for Radical Cystectomy in Bladder Cancer
}

\author{
Seung Hyun Jeon, M.D., Ph.D., Sung-Hyun Jeon, M.D. and Sung-Goo Chang, M.D., Ph.D. \\ Department of Urology, School of Medicine, Kyung Hee University, Seoul, Korea
}

Purpose: We investigated the effects of radical cystectomy and the prognostic factors that affect the survival of bladder cancer patients.

Materials and Methods: From 1979 to 2002, 59 patients with long-term follow up results of at least 2 years were enrolled in this study. Indications for surgery included muscle invasive bladder cancer and high-risk superficial bladder cancer. The cancer specific and recurrence free survival rates with respect to the possible prognostic factors were determined using Kaplan-Meier statistics.

Results: The mean patient age was 62.8 years (M: 48, $F:$ 11), and the estimated 5- and 10-year survival rates were $62 \%$ and $39.4 \%$, respectively. The median time to local or systemic recurrence was 16 months (range: 5 100 ), and the average survival durations after local and systemic recurrence were 14.4 months and 12.7 months, respectively. Pathologic stage, tumor grade, mean nuclear area, sex and lymphatic invasion were significant factors by univariate analysis $(p<0.05)$. The disease

\section{INTRODUCTION}

Bladder cancer has the highest incidence rate among the urologic cancers in Korea. In the 1990's, it was reported that 1500 new patients were registered annually with a diagnosis of bladder cancer (1). Bladder cancer is classified as superficial and invasive cancer; however, $75 \%$ of superficial tumors are recurrent and $10 \sim 15 \%$ progress on to become invasive tumors. Radical cystectomy remains the standard treatment for invasive bladder cancer that is confined to the bladder, but after surgery $50 \%$ of these patients show recurrence or distant metastasis,

Correspondence: Sung-Goo Chang, Department of Urology, School of Medicine, Kyung Hee University, 1 Hoegi-dong, Seoul 130-702 Korea. (Tel) 82-2-958-8533, (Fax) 82-2-959-6048, (E-mail) sgchang@ khu.ac.kr

Received November 22, 2005, Accepted January 11, 2005.

This work was supported by a grant from the Korean Science and Engineering Foundation, Republic of Korea (R13-2002-020-01001-0). related survival rate in patients having progression from an initial superficial tumor was lower than for those patients who displayed muscle invasive disease at the initial treatment. Multivariate analysis identified pathologic stage and lymphatic invasion as independent prognostic factors.

Conclusions: Radical cystectomy for organ-confined cancer showed favorable 5- and 10-year survival rates. The survival rate for patients with progression from an initial superficial tumor was worse than for those patients with invasive tumor at the initial presentation. The most significant independent prognostic factors were the pathologic stage and the presence of lymphatic invasion, which were highly correlated with all the investigated disease endpoints. (Cancer Research and Treatment 2005; $37: 48-53)$

Key Words: Radical cystectomy, Bladder cancer, Prognostic factor

and this leads to death in most cases within 2 years $(2,3)$. To improve the survival outcomes before or after cystectomy, various modalities such as radiation therapy, neoadjuvant or adjuvant combination chemotherapy have been attempted for patients who are considered at high risk of developing metastasis based on the pathologic findings at the time of cystectomy. Although the definitive roles of adjuvant or neoadjuvant chemotherapy in this setting have not been clarified, the results of small trials have been encouraging (4). Recent advances in organ-preserving procedures for invasive bladder cancer such as extensive transurethral resection, chemotherapy, radiotherapy and combined multimodal regimens have become popular because of the improved quality of life associated with retaining the native bladder $(5,6)$.

Various trials are being undertaken at this time to improve survival outcome, and we believed that the outcome of a contemporary cystectomy series with strict patient selection criteria would provide valuable information. Therefore, we retrospectively analyzed patients who had undergone radical cystectomy for preoperatively staged N0 and M0 bladder cancer, and we evaluated their long-term clinical outcome according to several clinical parameters to identify the possible independent prognostic factors. 


\section{MATERIALS AND METHODS}

\section{1) Patients}

Our retrospective study was performed on 59 patients, and their long-term follow up details exceeded 2 years. These 59 patients were chosen from among 75 patients who underwent radical cystectomy at Kyung Hee University Medical Center from 1979 to 2002 . Of these 59 patients, 48 were men $(81.4 \%)$ and 11 were women, and the median age of the total patients was 63 years (range: $45 \sim 81$ years). Indications for radical cystectomy were those patients who demonstrated muscle invasive bladder cancer that was confined to the bladder on the imaging studies, and those patients with superficial high-risk tumors such as multifocal, T1G3 and BCG-refractory CIS tumors. We also included the cases in which only the prostate urethra had been invaded. Urethrectomy was performed simultaneously in these cases. All the patients reported on in this study were preoperatively staged as N0 or M0 based on CT scan or MRI of the abdomen and pelvis, the IVP and the bone scan findings. Patients with non-TCC malignancy, gynecologic cancer patients and those patients with inadequate follow-up information were excluded. Moreover, even though we planned radical cystectomy based on the preoperative workup results, we did not proceed with surgery if the intraoperative frozen pelvic lymph node results were tumor positive.

\section{2) Surgical Technique:}

Lymphadenectomy. Bilateral pelvic lymphadenectomy was performed in all patients. The extent of lymphadenectomy included dissection of the obturator fossa only. Frozen section analysis was performed intraoperatively, and this procedure was abandoned if positive nodes were identified.

Radical cystectomy and urinary diversion. In the cases of prostatic urethra invasion, we performed a simultaneous urethrectomy. For the female patients, unless an orthotopic bladder substitution was performed, the procedure usually involves en bloc resection of the uterus together with most of the anterior wall of the vagina and the entire urethra. We constructed an ileal conduit in 54 patients and an orthotopic neobladder in 5 patients; the latter procedure has been performed since 1998 by using the sigmoid colon.

\section{3) Pathological findings:}

Cystectomy specimens. All en bloc cystectomy specimens were examined using the same pathological protocol. Pathologic staging of bladder tumor and lymph nodes was performed according to the 1997 TNM classification by the International Union Against Cancer and the American Joint Committee on Cancer. The pathologic subgroups were defined as organconfined $(\leq \mathrm{pT} 2)$ or non-organ-confined $(>\mathrm{pT} 2)$. In addition, we evaluated the lymphatic and vascular invasion that were located in the perivesical fat, and we assessed the prognostic importance of these findings.

The patients were also divided into three groups according to the histological results obtained from the initial and final transurethral tumor resections. In the first group, radical cystectomy was carried out for those patients having superficial bladder carcinoma with a high risk of progression. The second
Table 1. Characteristics of patients

\begin{tabular}{lrc}
\hline \multicolumn{1}{c}{ Characteristics } & No & $\%$ \\
\hline Pathologic staging & & \\
pTa-1/ pTIS* & & 15.3 \\
pT2 & 9 & 40.7 \\
pT3 & 24 & 28.8 \\
pT4 & 17 & 15.3 \\
Grade & 9 & \\
1 & & 11.9 \\
2 & 7 & 66.1 \\
3 & 39 & 22.0 \\
Tumor status at presentation & 13 & 23.7 \\
Superficial & 14 & 11.9 \\
Progressed from superficial & 7 & 64.4 \\
Initial invasive & 38 & \\
MNA $^{\dagger}$ (mean \pm SD) & $75.8 \pm 21(31.54 \sim 138.48)$ \\
\hline
\end{tabular}

*Transitional cell carcinoma in situ; ${ }^{\dagger}$ mean nuclear area.

group consisted of patients who displayed a superficial tumor at the first presentation and then they developed progressive muscle-invasive tumor while under conservative treatment. The third group of patients already had muscle-invasive disease at the first presentation.

Nuclear morphometry. All the resected specimens were fixed in $4 \%$ neutral buffered formalin and routinely embedded in paraffin wax using an automated tissue-processing unit. Histological sections (5 mm thick) were cut from each specimen, stained with haematoxylin and eosin and then assessed using an interactive image-analysis system comprised of a photomicroscope (Nikon, Tokyo, Japan) equipped with a high- resolution video camera (JVC, KKY-F-30, JCCD, Japan). For each patient, we randomly selected five microscope fields $(\times 400)$ for assessment: the nuclei of the tumor cells were outlined and measured, and we avoided the nuclei of stromal and necrotic areas. Fifty nuclei were measured for each patient and the mean nuclear area was calculated (square micrometers). The nuclear morphometric subgroups were defined as low $\left(<90 \mu^{2}\right)$ or high $\left(\geq 90 \mu^{2}\right)$.

\section{4) Statistics}

Overall survival and disease-free survival were estimated by using Kaplan-Meier curves. The log rank test was used to compare survival in the patient subgroups. In addition, univariate and multivariate analyses were performed using the Cox regression hazard model to evaluate the independent prognostic factors for the patients who underwent radical cystectomy. A p value of $<0.05$ was considered statistically significant.

\section{RESULTS}

\section{1) Patient characteristics and pathologic staging}

Among the 59 enrolled patients, organ-confined tumors ( $\leq$ pT2) were present in 33 patients (56\%): pTIS staged tumor was found in 1 patient, pT1 staged tumor was found in 8 patients, and pT2 staged tumor was found in 24 patients (Table 1). 

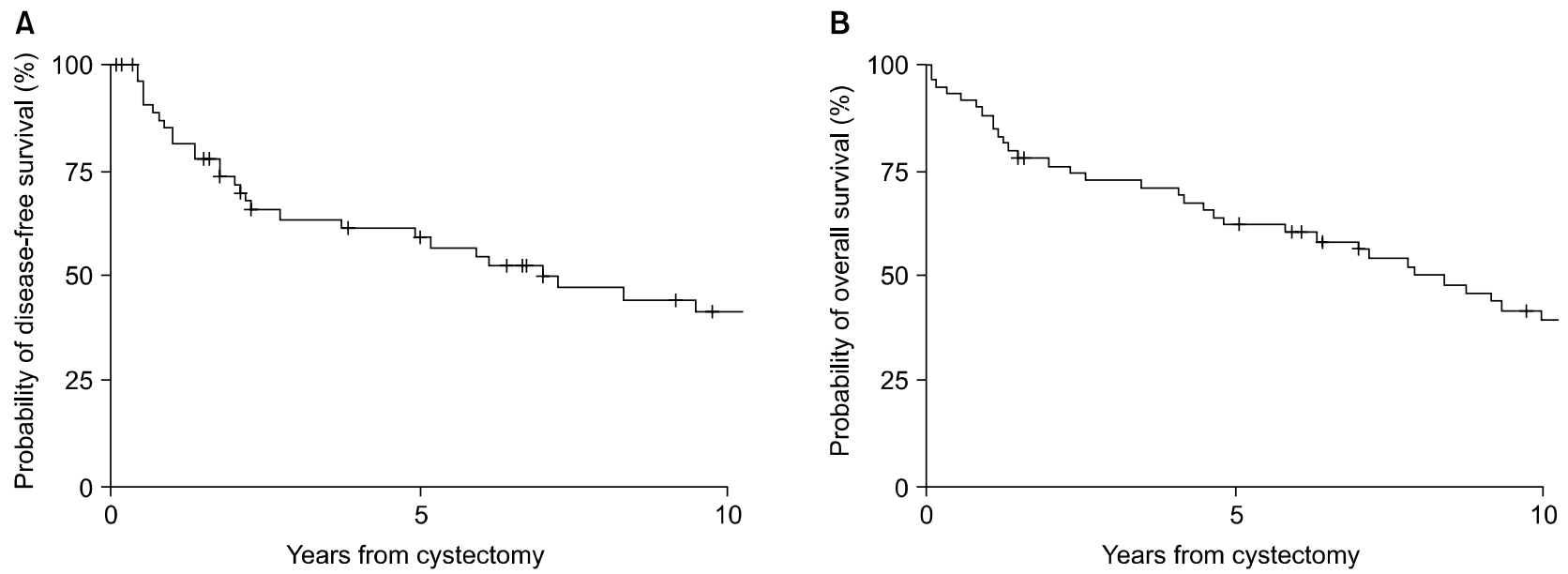

Fig. 1. Disease-free (A) and overall survival (B) for 59 patients that underwent radical cystectomy for bladder cancer.

A

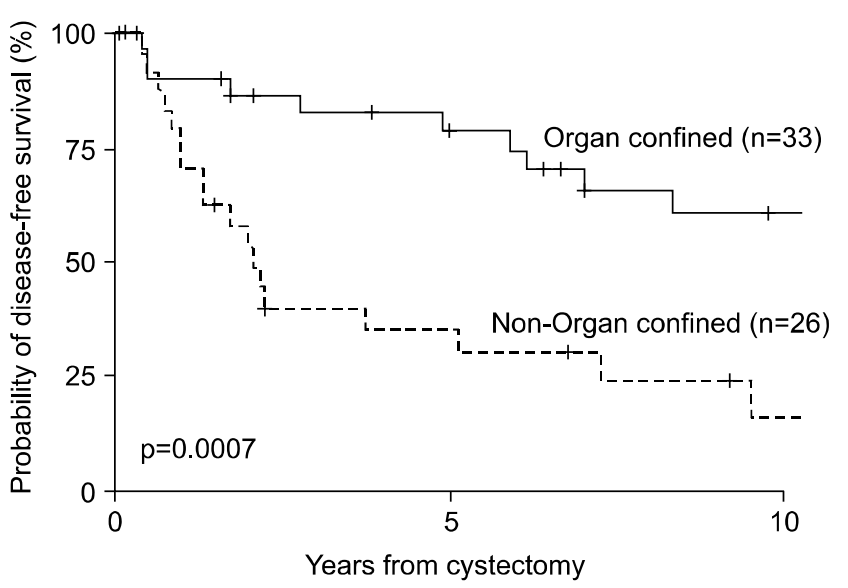

B

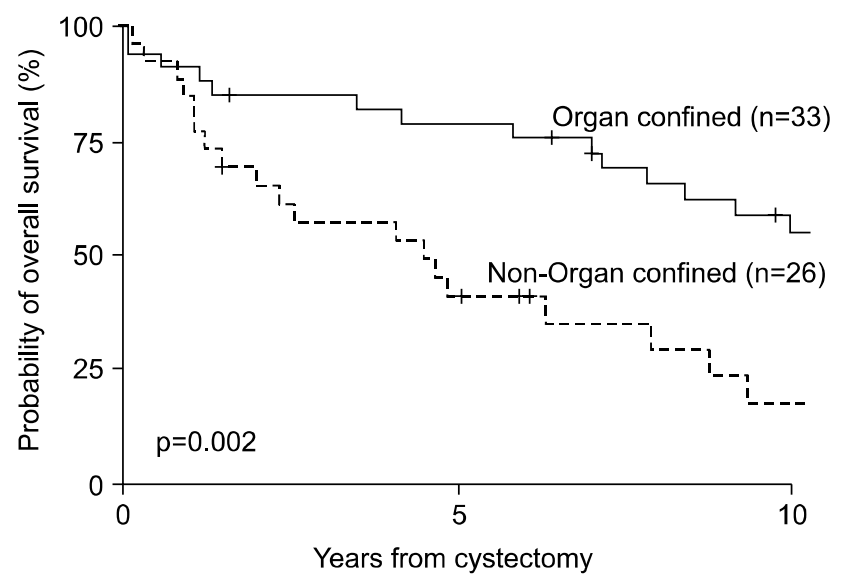

Fig. 2. Disease-free (A) and overall survival (B) for patients with organ-confined and non-organ-confined tumors.
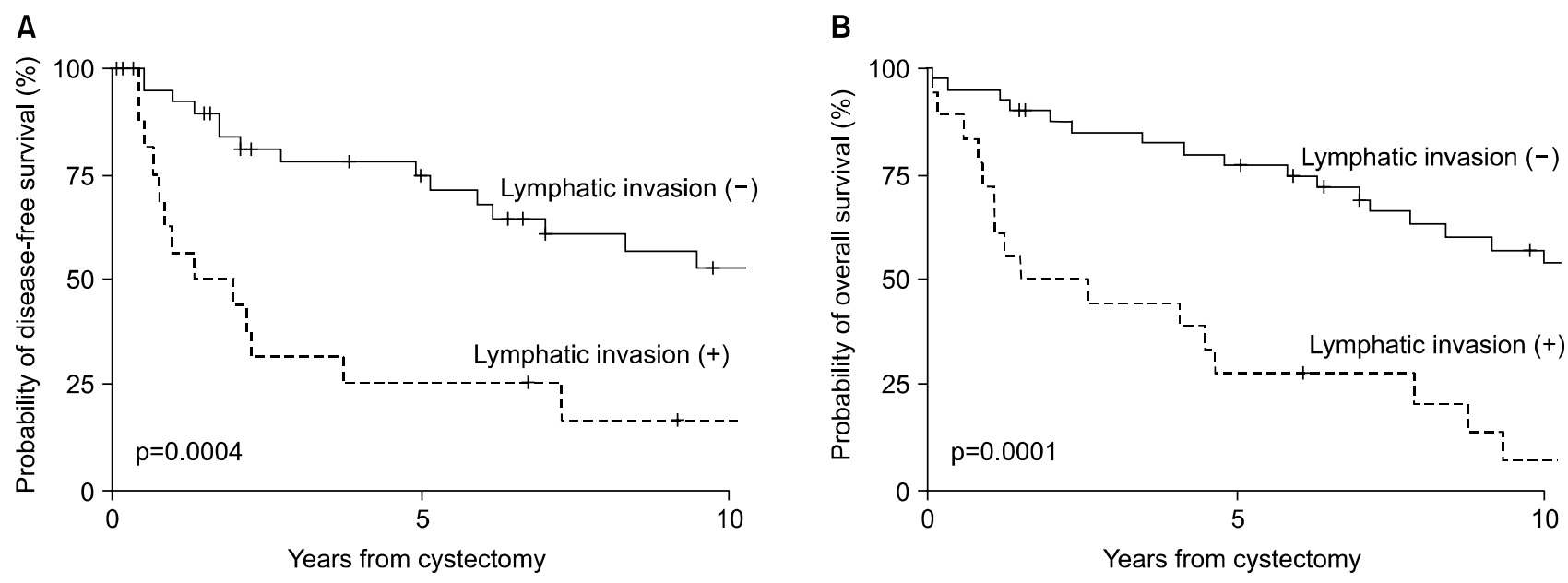

Fig. 3. Disease-free (A) and overall survival (B) for patients with and without lymphatic invasion. 
Table 2. Prognostic factors following radical cystectomy

\begin{tabular}{|c|c|c|c|c|}
\hline \multirow{2}{*}{ Prognostic factors } & \multicolumn{2}{|c|}{ Recurrence-free survival } & \multicolumn{2}{|c|}{ Overall survival } \\
\hline & $\mathrm{RR}(95 \% \mathrm{CI})$ & $\mathrm{p}$ value & $\mathrm{RR}(95 \% \mathrm{CI})$ & $\mathrm{p}$ value \\
\hline \multicolumn{5}{|l|}{ Univariate analysis: } \\
\hline Sex (male vs female) & $2.51(1.13 \sim 5.57)$ & 0.024 & $2.28(1.09 \sim 4.78)$ & 0.029 \\
\hline Organ confined vs non-organ confined & $3.57(1.63 \sim 7.84)$ & 0.001 & $2.67(1.4 \sim 5.1)$ & 0.003 \\
\hline Presence of lymphatic invasion (No vs Yes) & $3.54(1.67 \sim 7.53)$ & 0.001 & $3.33(1.73 \sim 6.43)$ & 0.000 \\
\hline Presence of vascular invasion (No vs Yes) & $0.33(0.11 \sim 1.9)$ & 0.277 & $0.45(0.08 \sim 1.4)$ & 0.132 \\
\hline \multicolumn{5}{|l|}{ Tumor Grade } \\
\hline Grade 1 & 1 & & 1 & \\
\hline Grade 2 & $4.46(0.59 \sim 33.59)$ & 0.146 & $2.46(0.74 \sim 8.12)$ & 0.141 \\
\hline Grade 3 & $14.64(6.86 \sim 25.42)$ & 0.011 & $4.82(1.31 \sim 17.69)$ & 0.018 \\
\hline MNA $\left(<90\right.$ vs $\left.\geq 90 \mu \mathrm{m}^{2}\right)$ & $1.99(0.94 \sim 4.25)$ & 0.072 & $2.59(1.34 \sim 5.02)$ & 0.005 \\
\hline \multicolumn{5}{|l|}{ Tumor status at presentation } \\
\hline Superficial & 1 & & 1 & \\
\hline Progressed from superficial & $9.07(2.2 \sim 37.37)$ & 0.002 & $5.59(1.84 \sim 16.99)$ & 0.002 \\
\hline Initial invasive & $2.91(1.37 \sim 9.03)$ & 0.04 & $1.93(1.52 \sim 6.81)$ & 0.017 \\
\hline \multicolumn{5}{|l|}{ Multivariate analysis } \\
\hline Organ confined vs non-organ confined & $2.44(1.25 \sim 4.78)$ & 0.009 & $4.69(1.73 \sim 12.73)$ & 0.002 \\
\hline Presence of lymphatic invasion (No vs Yes) & $3.67(1.65 \sim 8.14)$ & 0.001 & $3.2(1.65 \sim 6.21)$ & 0.001 \\
\hline
\end{tabular}

Univariate analysis by Cox proportional hazards model and final Cox multiple regression model after forward conditional variable selection.

Non-organ-confined tumors $(>\mathrm{pT} 2)$ were found in 26 patients (44\%): pT3 staged tumor was found in 17 patients and pT4 staged tumor was found in 9 patients (Table 1). Despite the negative preoperative staging in all patients, the final pathology demonstrated positive perivesical lymphatic invasion in 18 patients $(30.5 \%)$ and vascular invasion in 3 patients $(5.1 \%)$ The incidences of lymphatic invasion were $12.5 \%$ in the pT2 staged patients, $58.8 \%$ in the pT3 staged patients and $44.4 \%$ in the pT4 staged patients.

According to the histologic results of the initial and final transurethral resections of bladder tumor (TURBTs), 14 patients (23.7\%) underwent radical cystectomy for a superficial bladder carcinoma that had a high likelihood of progressing. Seven patients $(11.9 \%)$ presented with a superficial tumor at the first TURBT, but later they eventually developed a progressive muscle-invasive tumor while under conservative management (Table 1), and another 38 patients $(64.4 \%)$ had muscle invasive disease at the time of the first TURBT.

\section{2) Overall Survival and Disease-free survival}

The median follow-up of the 59 patients was 77 months (mean follow-up: 88 months; range: 1 to 272 months). A total of 3 patients $(5.1 \%)$ died within 3 months of their surgery, and the reasons for death were all non-tumor-related (i.e., panperitonitis for 2 patients, and accidental death for 1 patient). The recurrence-free survival rate and overall survival rate for all the patients at 5 years were $59.3 \%$ and $62 \%$, respectively, and $41.3 \%$ and $39.4 \%$, respectively, at 10 years (Fig. 1). Five-year overall survival was reduced according to tumor staging, from $88.8 \%$ for patients with pT1 tumors, to $75 \%$ for those with pT2 tumors, to $47 \%$ for those with pT3 tumors and to $25.9 \%$ for those with pT4 tumors. Disease-free survival and overall survival were closely correlated with the primary tumor stage and with the presence of lymphatic invasion (Fig 2, 3)

In addition to the pathological stage and lymphatic invasion, recurrence-free survival and overall survival were also found to be closely correlated with gender, mean nuclear area (MNA), tumor grade and the type of disease at presentation by univariate analysis $(p<0.05)$ (Table 2 ).

It is interesting that survival rates were clearly depended on the disease type at the time of presentation. The 5-year overall survival rates for patients with superficial tumor, initial invasive tumor, and of those patients that progressed from an initial superficial tumor were $84.4 \%, 64.8 \%$ and $16.7 \%$ respectively. In particular, the lower survival rate of patients who underwent cystectomy because of progression from an initial superficial tumor at the first TURBT as compared with those patients who displayed muscle invasive disease at first TURBT was noteworthy.

By multivariate analysis, the pathologic stage and lymphatic invasion were found to be significant independent factors of overall and disease-free survival (Table 2).

\section{3) Local recurrence versus distant metastasis}

Tumor progression was observed in 26 of the $59(44 \%)$ patients. The median time to progression in these 26 patients was 16 months (range: 5 to 100 months). Local recurrence was observed in $13(22 \%)$ patients and distant metastases were observed in 19 patients $(32.2 \%)$. The sites of local recurrence were mainly the prostate, pelvic cavity, inguinal area, penis and anus, and the incidence of local and distant failures was found to be closely correlated with the pathologic stage (Table 3 ). The incidence of local recurrence increased from $11.1 \%$ for patients with pT1 tumors, to $20.8 \%$ for patients with pT2 tumor, $23.5 \%$ for patients with pT3 tumor, and $33.3 \%$ for patients with pT4 tumor. Likewise, the incidence of distant metastasis increased 
Table 3. Local recurrence and distant failure according to tumor stage

\begin{tabular}{lcccccc}
\hline \multirow{2}{*}{ Tumor stage } & \multicolumn{2}{c}{ Local recurrence } & \multicolumn{2}{c}{ Distant failure } \\
\cline { 2 - 3 } \cline { 6 - 7 } & & No & $\%$ & & No & $\%$ \\
\hline pTa-T1/TIS* $(\mathrm{n}=9)$ & 1 & 11.1 & & 1 & 11.1 \\
pT2 $(\mathrm{n}=24)$ & 5 & 20.8 & & 7 & 29.2 \\
pT3 $(\mathrm{n}=17)$ & 4 & 23.5 & & 6 & 35.3 \\
pT4 $(\mathrm{n}=9)$ & 3 & 33.3 & & 5 & 55.6 \\
Organ confined $(\mathrm{n}=33)$ & 6 & 18.2 & & 8 & 24.2 \\
Non-organ confined $(\mathrm{n}=26)$ & 7 & 26.9 & & 11 & 42.3 \\
Lymphatic invasion $(-)(\mathrm{n}=41)$ & 8 & 19.5 & & 10 & 24.4 \\
Lymphatic invasion $(+)(\mathrm{n}=18)$ & 5 & 27.7 & & 9 & 50 \\
\hline
\end{tabular}

*Transitional cell carcinoma in situ.

from $11.1 \%$ for patients with pT1 tumors, to $29.2 \%$ for patients with pT2 tumor, to $35.3 \%$ for patients with pT3, and to $55.6 \%$ for patients with pT4 tumor (Table 3 ). The average survival duration after local recurrence was 14.4 months and the average survival duration after systemic recurrences was 12.7 months.

\section{4) Complications of radical cystectomy}

Thirteen patients (22\%) and 9 patients (15.3\%) sustained early or late complications, respectively. The most frequent complications were urine leakage $(10.2 \%)$ during the early postoperative period and stomal stenosis $(5.1 \%)$ in the late postoperative period. Two patients that suffered from bowel leakage died within the first postoperative month because of panperitonitis and sepsis despite that they underwent re-operation.

\section{DISCUSSION}

Radical cystectomy remains the standard treatment for invasive bladder cancer, and this operation is also performed for recurring tumors and for poor differentiated multifocal superficial bladder cancer. The pathologic stage of primary bladder tumor, the pathologic subgroups and lymph node involvement are the important survival determinants (7). However, the preoperative clinical staging and TUR staging tend to be inadequate in this content. Pagano et al (8) have reported that the over-all staging errors in terms of differentiating the clinical and pathologic stages were as high as $44 \%$, and that $35 \%$ of stage $\mathrm{T} 1$ cancers were understaged whereas Bosl (9) reported that $39 \%$ of $\mathrm{T} 2$ staged patients were understaged. Similarly, in the present study, when excluding the 2 cases that were incompletely resected during TURBT, 2 of the 11 patients $(18.2 \%)$ who were classified as stage T1 at TURBT and 6 of 44 patients $(13.6 \%)$ who were classified as stage T2 were eventually found to be understaged.

Our study supports the role of radical surgery for invasive bladder cancer: the recurrence-free survival rate at 5 years was $59.3 \%$ for the entire study group and $78.8 \%$ for the patients with organ-confined tumors. A local recurrence rate of $26.9 \%$ and a distant failure rate of $42.3 \%$ for non-organ confined tu mors are of extreme significance for accurate preoperative staging. However, the preoperative clinical staging systems have a critical limitation, and that is that microscopic metastases cannot be found. In our series, 8 patients $(30.5 \%)$ showed postoperative lymphatic invasion, and they experienced a consequent miserable survival outcome of $31 \%$ and $25 \%$ for the 5year overall survival rate and disease-free survival rate, respectively, despite of the negative preoperative imaging studies.

This analysis of patients with a long-term follow-up enabled us to identify the important prognostic variables that could be used to stratify patients after radical cystectomy. The pathological variables of primary bladder tumor and the presence of lymphatic invasion were identified as the important and independent prognostic variables of overall and disease-free survival for patients that underwent radical cystectomy. Univariate analysis showed survival differences according to gender and the MNA subgroup. Many researchers have considered the MNA to be a possible predictor of superficial bladder cancer progression, and they have suggested that it be used to complement the grading systems as a quantitative biomarker $(10,11)$. However, it's still mandatory that its prognostic significance be further examined in independent patient sets. Interestingly, our data show that female patients had a poorer survival than the male patients (5-year overall survival; $36.3 \%$ in women vs $72 \%$ in men, 5-year disease free survival; $36.3 \%$ in women vs. $65.6 \%$ in men). There were no significant differences found between the distributions of the pathologic stages in males and females. In women, T1 tumor was found in 1 patient $(9.1 \%)$, $\mathrm{T} 2$ tumor was found in 5 patients $(45.5 \%)$, and $\mathrm{T} 3$ tumor was found in 5 patients $(45.5 \%)$, whereas in the men, T1 tumor was found in 8 patients (16.7\%), T2 tumor was found in 19 patients $(39.6 \%), \mathrm{T} 3$ tumor was found in 12 patients $(25 \%)$, and $\mathrm{T} 4$ tumor was found in 9 patients (18\%). Some researchers have suggested that the female survival rate is lower because of a larger tumor size, a poorer general condition or a more advanced tumor state at the time of discovery $(12,13)$. However, the prognostic role of gender for bladder cancer remains to be determined.

Another noteworthy result of the present study was the survival difference observed between patients with an invasive tumor at the first TURBT and those patients with a superficial tumor at the first TURBT that later became invasive. Several studies have reported that the prognosis of superficial tumor that progresses despite of conservative management is no better than that of invasive TCC found at the initial presentation (14, 15). The reason for the lower survival rate of patients showing invasive progression from superficial disease could be related to late discovery of tumor and the partial follow-up loss of patients. Actually, radical cystectomy specimens in the 7 patients affected revealed T2 tumor in 2 patients, T3 tumor in 2 patients and T4a tumor in 3 patients. Five of them were diagnosed because of hematuria after a follow-up loss period. The median interval between the initial TURBT and cystectomy was 51 months. On the other hand, the 5-year disease-free survival rate and disease-free survival rate for patients that underwent cystectomy at the superficial stage were $88.8 \%$ and $80.8 \%$ respectively. These data support the notion that early cystectomy for high-risk superficial tumors much improves the survival results. 
The limitations of this retrospective study include its small sample size and the possibility of a selection bias that influenced the survival outcome. Prospective studies involving a larger series are necessary to confirm our findings.

\section{CONCLUSIONS}

Radical cystectomy for patients with organ-confined bladder cancer was found to provide an excellent 5-year survival of $82.3 \%$. The poor survival results of patients with tumors that progressed from an initial superficial cancer support the use of early cystectomy for patients with a high-risk superficial tumor. Our study shows that the pathologic staging and lymphatic invasion are the most important prognostic factors for both overall survival and disease-free survival for patients that have undergone radical cystectomy for bladder cancer. The roles of sex, MNA and tumor grade as prognostic factors remain to be elucidated.

\section{REFERENCES}

1. Lee C, Lee ES, Choi H, Koh SK, Lee JM, Chai SE, et al. Incidence estimation of genitourinary cancer in Korea. J Korean Med Sci. 1992;7:154-61.

2. Prout GR Jr, Griffin PP, Shipley WU. Bladder carcinoma as a systemic disease. Cancer. 1979;43:2532-9.

3. Babaian RJ, Johnson DE, Llamas L, Ayala AG. Metastases from transitional cell carcinoma of the urinary bladder. Urology. 1980;16:142-4.

4. Skinner DG, Daniels JR, Russell CA, Lieskovsky G, Boyd SD, Nichols $\mathrm{P}$, et al. The role of adjuvant chemotherapy following cystectomy for invasive bladder cancer: a prospective com- parative trial. J Urol. 1991;145:459-64.

5. Herr HW. Transurethral resection of muscle-invasive bladder cancer: 10-year outcome. J Clin Oncol. 2001;19:89-93.

6. Sternberg CN. Current perspectives in muscle invasive bladder cancer. Eur J Cancer. 2002;38:460-7.

7. Stein JP, Lieskovsky G, Cote R, Groshen S, Feng AC, Boyd $\mathrm{S}$, et al. Radical cystectomy in the treatment of invasive bladder cancer: long-term results in 1,054 patients. J Clin Oncol. 2001; 19:666-75.

8. Pagano F, Bassi P, Galetti TP, Meneghini A, Milani C, Artibani $\mathrm{W}$, et al. Results of contemporary radical cystectomy for invasive bladder cancer: a clinicopathological study with an emphasis on the inadequacy of the tumor, nodes, and metastases classification. J Urol. 1991;145:45-50.

9. Bosl GJ. Controversies in the management of transitional cell carcinoma of the bladder. In: American Society of Clinical Oncology Educational Book 1991:73-88.

10. Bol MG, Baak JP, Rep S, Marx WL, Kruse AJ, Bos SD, et al. Prognostic value of proliferative activity and nuclear morphometry for progression in TaT1 urothelial cell carcinomas of the urinary bladder. Urology. 2002;60:1124-30.

11. Ozer E, Yorukoglu K, Mungan MU, Ozkal S, Demirel D, Sagol O, et al. Prognostic significance of nuclear morphometry in superficial bladder cancer. Anal Quant Cytol Histol. 2001; 23:251-6.

12. Moran A, Sowerbutts AM, Collins S, Clarke N, Cowan R. Bladder cancer: worse survival in women from deprived areas. Br J Cancer. 2004;90:2142-44.

13. Narayana AS, Loening SA, Sylmen DJ, Culp DA. Bladder cancer: factors affecting survival. J Urol. 1983;130:56-60.

14. May M, Helke C, Nitzke T, Vogler H, Hoschke B. Survival rates after radical cystectomy according to tumor stage of bladder carcinoma at first presentation. Urol Int. 2004;72:103-11.

15. Yiou R, Patard JJ, Benhard H, Abbou CC, Chopin DK. Outcome of radical cystectomy for bladder cancer according to the disease type at presentation. BJU Int. 2002;89:374-8. 